\title{
Two Cases of Nivolumab Re-Administration after Pneumonitis as Immune-Related Adverse Events
}

\author{
Keisuke Imafuku ${ }^{a} \quad$ Koji Yoshino ${ }^{a} \quad$ Kei Yamaguchi ${ }^{a}$ Satoshi Tsuboi ${ }^{a}$ \\ Kuniaki Ohara $^{a}$ Hiroo Hata $^{b}$ \\ ${ }^{a}$ Tokyo Metropolitan Cancer and Infectious Diseases Center, Komagome Hospital, \\ Tokyo, Japan; ${ }^{b}$ Department of Dermatology, Hokkaido University Graduate School of \\ Medicine, Sapporo, Japan
}

\section{Keywords}

Nivolumab · Immune-related adverse event · Re-administration · Unresectable malignant melanoma $\cdot$ Cryptogenic organizing pneumonia $\cdot$ Diffuse alveolar damage $\cdot$ Pneumonitis

\begin{abstract}
Nivolumab is a recently approved medication for the treatment of unresectable malignant melanoma. Many immune-related adverse events (irAEs) associated with nivolumab have been reported, such as pneumonitis, hepatitis, dermatitis, and thyroiditis. Prednisolone can effectively treat irAEs. However, it is unclear how or if nivolumab should be administered to patients after they have experienced an irAE. Herein, we show 2 patients who underwent pneumonitis as irAE. Case 1 demonstrated a cryptogenic organizing pneumonia pattern in the CT scan and case 2 had a diffuse alveolar damage (DAD) pattern. Oral corticosteroids improved chest shadow of CT scan in both cases. However, when nivolumab was re-administrated, case 1 demonstrated no symptoms, but case 2 demonstrated pneumonia again. From our cases, it is difficult to re-administrate nivolumab for the patients with pneumonitis which shows a DAD pattern in $C T$, even if oral corticosteroids improve their symptoms.




\section{Case Reports in Oncology}

\section{Introduction}

Antibodies against programmed cell death-1 (PD-1) are newly approved medications for the treatment of unresectable malignant melanoma. Nivolumab, a PD-1 inhibitor, improves overall survival among melanoma patients compared to conventional therapy, such as dacarbazine [1]. However, treatment with nivolumab is associated with a variety of immunerelated adverse events (irAEs) [2]. Anti PD-1 agents, such as nivolumab, cause fewer irAEs compared to CTLA-4 inhibitors (e.g., ipilimumab), and the clinical benefits appear to be superior to ipilimumab $[2,3]$. IrAEs due to immune system inhibitors are very different from toxicities observed with conventional cytotoxic chemotherapy. The possible reactions include the development of rashes, vitiligo, colitis, interstitial pneumonia (IP), hepatitis, thyroiditis, nephritis, and hypophysitis [4]. Initial therapies for irAEs in both ipilimumab and nivolumab have been proposed in some literatures. However, it is actually difficult to decide to re-administrate nivolumab after a patient has recovered from an irAE. In our institution, 27 patients have received nivolumab treatment, and some patients experienced severe irAEs, such as hepatitis, colitis, and pneumonitis. Nivolumab was discontinued, and the patients improved following treatment with systemic corticosteroids. However, it was unclear whether to resume nivolumab therapy. Nevertheless, we decided to resume nivolumab therapy after obtaining informed consent from the patients. Today, the treatment of irAE seems to be a current topic for oncologists. Since no studies have evaluated the effects of nivolumab re-administration following an irAE episode, we present the results from 2 cases of nivolumab re-administration to facilitate the development of guidelines for managing this clinical scenario.

\section{Patients}

Patients were enrolled from 2014 to 2015 at the Tokyo Metropolitan Cancer and Infectious Diseases Center of the Komagome Hospital. The following patient data were collected: age, sex, primary lesion information (see Table 1 for a complete list of patient characteristics). A molecular investigation revealed the absence of the BRAF V600E mutation in all patients. In all cases, nivolumab was administered every 3 weeks at a dose of $2 \mathrm{mg} / \mathrm{kg}$, which differs from the dosage approved by the FDA.

\section{Case Presentations}

Case 1

A 62-year-old male with digital melanoma was treated with nivolumab for bone metastasis. After 6 courses of nivolumab, the patient became febrile to $38^{\circ} \mathrm{C}$ and complained of mild respiratory discomfort. A repeat CT scan revealed bilateral lower lobe lung consolidations that were consistent with cryptogenic organizing pneumonia (COP) pattern of nivolumab-induced IP (grade 1) (Fig. 1a). Therefore, systemic corticosteroid therapy was administered ( $1 \mathrm{mg} / \mathrm{kg} /$ day), and the dose was gradually reduced as the lung lesions im- 


\section{Case Reports in Oncology}

Case Rep Oncol 2017;10:296-300

DOI: $10.1159 / 00046337$

(C) 2017 The Author(s). Published by S. Karger AG, Base www.karger.com/cro

Imafuku et al.: Two Cases of Nivolumab Re-Administration after Pneumonitis as Immune-Related Adverse Events

proved (Fig. 1b). The patient was then re-treated with nivolumab and oral corticosteroids $(0.15 \mathrm{mg} / \mathrm{kg} /$ day) without any further evidence of IP.

Case 2

A 75-year-old male with rectal melanoma was referred to our hospital. The patient presented with bone, lung, and liver metastases. We began treatment with nivolumab for unresectable melanoma. Four weeks following the first administration of nivolumab, the patient suddenly developed difficulty in breathing. A CT scan revealed widespread bilateral groundglass opacity of his lungs consistent with a diffuse alveolar damage (DAD) pattern (grade 2) (Fig. 1c). He was treated with systemic corticosteroids $(1 \mathrm{mg} / \mathrm{kg} / \mathrm{day})$ for nivolumabinduced IP. Following improvement of his respiratory status (Fig. 1d), nivolumab therapy was resumed with oral corticosteroids $(0.25 \mathrm{mg} / \mathrm{kg} / \mathrm{day})$. However, 3 days later, he experienced a sudden exacerbation of his respiratory status. A repeat CT scan revealed recurrent nivolumab-induced IP (Fig. 1e). Steroid pulse therapy with 1,000 mg of methylprednisolone was initiated.

\section{Discussion}

IrAEs due to PD-1 inhibitors are mainly grade 1 or 2 in severity and can be managed with algorithms developed for irAEs associated with ipilimumab [4, 5]. General principles for the optimal management of irAEs include early recognition and correct use of immunosuppressive agents based on the severity of the episode. IrAEs are reversible with prompt recognition. However, failing to manage these cases appropriately can lead to severe toxicity and death [6]. Treatment for irAEs generally consists of the administration of immunosuppressive agents, such as corticosteroids or other agents such as TNF- $\alpha$ inhibitors [4]. Although re-administration of nivolumab is allowed in grade1-2 irAE, it is often abandoned in grade 3-4. With the consent of the patients, we re-administered nivolumab to 2 patients after the irAEs improved because of a lack of alternative therapy such as vemurafenib at that time. In case 2, pneumonitis was severe and diffusely spread throughout both lungs. Nonetheless, corticosteroid therapy was effective, and the pneumonitis improved. Therefore, the patient was re-administered nivolumab with corticosteroids.

In our cases, IP was more severe than colitis and required more time to respond to treatment. Because it is not desirable to discontinue therapy in cases of advanced melanoma, we attempted to resume nivolumab therapy early along with corticosteroids to prevent irAE recurrence. Based on the clinical responses, it seems feasible to re-administer nivolumab without corticosteroids in some cases. However, in cases of pneumonitis, great care must be taken because of the possibility of recurrent IP even with concurrent corticosteroid use. There are several IP patterns. Based on these classifications [7], case 1 demonstrated a pattern consistent with COP. The pattern in case 2 was consistent with DAD. In general, COP is more responsive to treatment with corticosteroids than DAD. Eventually, we do not recommend re-initiating treatment with nivolumab in patients with pneumonitis that is consistent with DAD according to a CT scan. In particular, the second CT scan in case 2 revealed severe recurrent pneumonitis. Therefore, we decided to begin steroid pulse therapy. 
When both the occurrence and improvement of irAEs were rapid compared to what is usually observed, it is feasible to re-administer nivolumab with a rapid dose reduction of corticosteroids. However, this is not a desirable approach in our cases of IP, even with the continued use of corticosteroids. Particularly, for cases of IP that are consistent with a DAD pattern, we suggest that the corticosteroid dose is gradually reduced over time, and nivolumab should be discontinued (replaced with another agent). We report 2 cases of nivolumab re-administration after irAEs. The occurrence of IP is associated with a slow or incomplete response and is likely to recur following nivolumab re-administration in combination with corticosteroids, particularly in patients with IP (DAD pattern). In this report, we recommend that in the event of an irAE, corticosteroids are administered first. The occurrence of IP is associated with a slow or incomplete response and is likely to recur following nivolumab re-administration in combination with corticosteroids, particularly in patients with IP (DAD pattern).

\section{Statement of Ethics}

The authors have no ethical conflicts to disclose.

\section{Disclosure Statement}

The authors have no conflicts of interest to disclose.

\section{Funding Sources}

None.

\section{References}

1 Robert C, Long GV, Brady B, et al: Nivolumab in previously untreated melanoma without BRAF mutation. N Engl J Med 2015;372:320-330.

2 Topalian SL, Hodi FS, Brahmer JR, et al: Safety, activity, and immune correlates of anti-PD-1 antibody in cancer. N Engl J Med 2012;366:2443-2454.

-3 Hamid O, Robert C, Daud A, et al: Safety and tumor responses with lambrolizumab (anti-PD-1) in melanoma. N Engl J Med 2013;369:134-144.

4 Weber JS, Kahler KC, Hauschild A: Management of immune-related adverse events and kinetics of response with ipilimumab. J Clin Oncol 2012;30:2691-2697.

5 Fecher LA, Agarwala SS, Hodi FS, et al: Ipilimumab and its toxicities: a multidisciplinary approach. Oncologist 2013;18:733-743.

Villadolid J, Amin A: Immune checkpoint inhibitors in clinical practice: update on management of immune-related toxicities. Transl Lung Cancer Res 2015;4:560-575.

7 American Thoracic Society/European Respiratory Society International Multidisciplinary Consensus Classification of the Idiopathic Interstitial Pneumonias. This joint statement of the American Thoracic Society (ATS), and the European Respiratory Society (ERS) was adopted by the ATS board of directors, June 2001 and by the ERS Executive Committee, June 2001. Am J Respir Crit Care Med 2002;165:277304. 


\section{Case Reports in Oncology}

\begin{tabular}{|c|c|}
\hline DOI: $10.1159 / 000463379$ & $\begin{array}{l}\text { (C) } 2017 \text { The Author(s). Published by S. Karger AG, Basel } \\
\text { www.karger.com/cro }\end{array}$ \\
\hline
\end{tabular}

Imafuku et al.: Two Cases of Nivolumab Re-Administration after Pneumonitis as Immune-Related Adverse Events
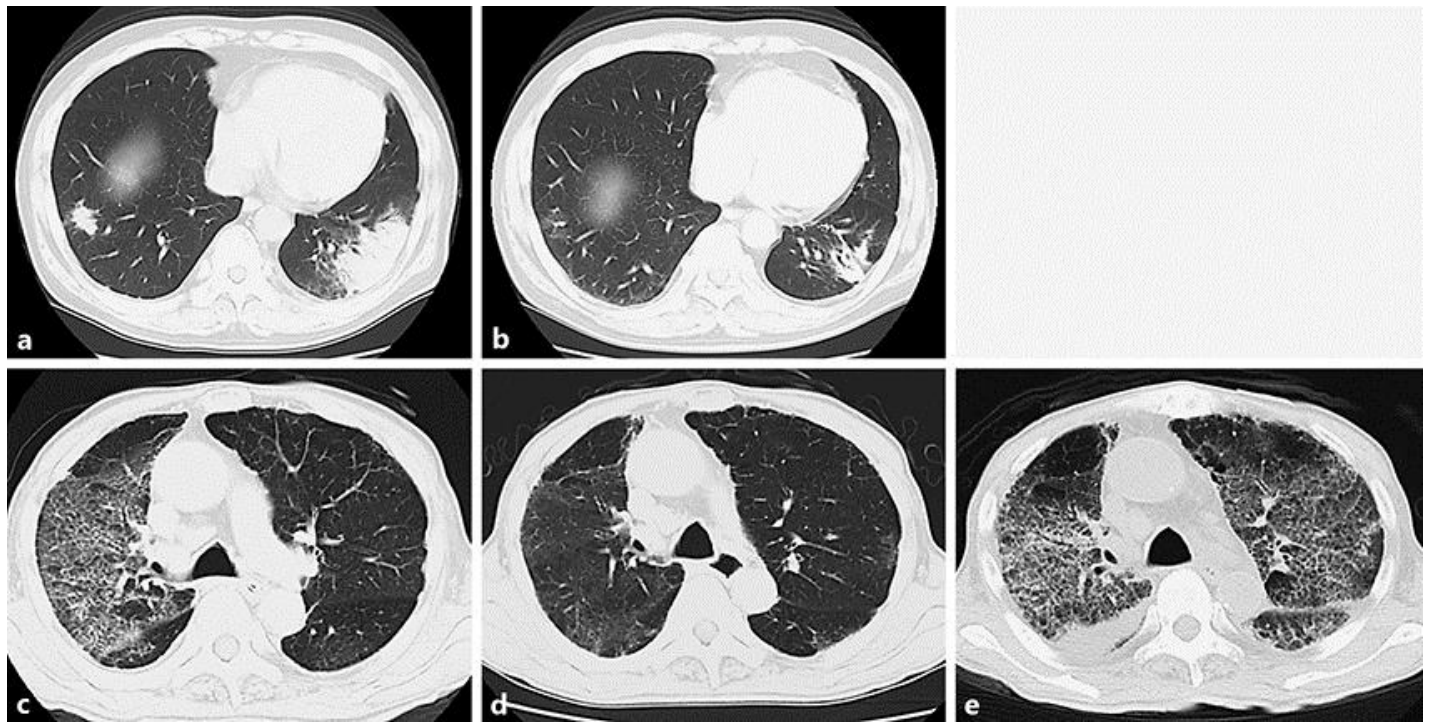

Fig. 1. CT scans of both cases. a In case 1, a focal patch of subpleural consolidation was observed after nivolumab re-administration. b Pneumonitis gradually improved with corticosteroids. c In case 2, diffuse ground glass opacity was observed in the lungs. $\mathbf{d}$ Pneumonitis had clearly improved. e Diffuse bilateral pneumonitis recurred in the lungs after nivolumab re-administration.

Table 1. Details of the patients

\begin{tabular}{|c|c|c|c|c|c|c|c|c|}
\hline Case & $\begin{array}{l}\text { Age, } \\
\text { years }\end{array}$ & Sex & $\begin{array}{l}\text { Primary } \\
\text { lesion }\end{array}$ & $\begin{array}{l}\text { Metastatic } \\
\text { site }\end{array}$ & $\begin{array}{l}\text { CT } \\
\text { pattern }\end{array}$ & Treatment for irAE & $\begin{array}{l}\text { Interval to } \\
\text { re-administration }\end{array}$ & $\begin{array}{l}\text { Recurrence } \\
\text { of irAE }\end{array}$ \\
\hline 1 & 62 & M & Finger & $\begin{array}{l}\text { Bone } \\
\text { Brain }\end{array}$ & $\mathrm{COP}$ & PSL $1.0 \mathrm{mg} / \mathrm{kg} /$ day & 5 weeks & - \\
\hline 2 & 75 & M & Rectum & $\begin{array}{l}\text { Lungs } \\
\text { Liver } \\
\text { Bone }\end{array}$ & DAD & PSL $1.0 \mathrm{mg} / \mathrm{kg} /$ day & 9 weeks & - \\
\hline
\end{tabular}

COP, cryptogenic organizing pneumonia; DAD, diffuse alveolar damage; irAE, immune-related adverse event. 